\title{
Modified jump conditions for anisotropic temperature plasmas at parallel shocks
}

\author{
M. Siewert and H.-J. Fahr \\ Argelander Institut für Astronomie der Universität Bonn, Abteilung für Astrophysik und Extraterrestrische Forschung, \\ Auf dem Huegel 71, 53121 Bonn, Germany \\ e-mail: msiewert@astro.uni-bonn.de
}

Received 16 October 2008 / Accepted 27 March 2009

\section{ABSTRACT}

\begin{abstract}
Aims. We reinvestigate the parallel pressure-anisotropic MHD shock with an emphasis on the downstream stability of the plasma with respect to the firehose instability.

Methods. Aiming to close the underdetermined jump conditions, we take advantage of a kinetic model of the shock transit, based on the Boltzman-Vlasow equation with CGL invariants. In addition, we show that we need to consider plasma waves as an additional component of the system.

Results. The system of jump conditions and the kinetic model reduces to a relatively simple form. At first glance, about all parallel magnetosonic shocks must be considerd unstable, at least with respect to plasma-wave generation caused by the firehose instability. In a similar way to this fundamental instability, the system will be also unstable when considering other plasma instabilities.
\end{abstract}

Key words. plasmas - shock waves - magnetohydrodynamics (MHD) - solar wind

\section{Introduction}

In the past years, in-situ measurements of shock waves in astrophysical plasma systems, such as planetary bow shocks or the solar wind termination shock, have greatly refined our knowledge of the microphysics of these MHD boundary layers. A few years ago, the spacecraft Voyager-1 (Burlaga et al. 2005; Decker et al. 2005; Gurnett \& Kurth 2005; Stone et al. 2005) and Voyager-2 (Richardson et al. 2008; Decker et al. 2008; Stone et al. 2008; Burlaga et al. 2008; Gurnett \& Kurth 2008) both crossed the solar wind termination shock; the first in December 2004 (Fisk 2005) at a distance of 97 AU and the second in September 2007 at a distance of 86 AU (Jokipii 2008). These crossing events provided detailed data on the fine structure of these heliospheric transition layers.

However, none of the data taken are in full agreement with earlier theoretical model predictions based on the classical MHD jump conditions (see e.g. Gombosi 1998; Diver 2001). For example, Voyager-1 found an unexpectedly low mass density compression ratio of $x_{\mathrm{VOY}-1}=\rho_{2} / \rho_{1} \simeq 2.5$, strong compressive magnetic fluctuations downstream of the shock (Burlaga et al. 2006), in addition to the unexpected fact that the spectral intensities of the anomalous cosmic ray (ACR) component, at least at higher energies above $10 \mathrm{MeV} /$ nucleon, do not peak at the shock as expected, but instead continue to increase further downstream (Kiraly 2005; Stone et al. 2005). In contrast to this, Voyager- 2 crossed the termination shock at a completely different position, observing the expected plateau in the ACR intensity on the downstream side of the shock (Stone et al. 2008). On the other hand, this spacecraft encountered the boundary layer approximately 10 AU closer to the Sun than expected, with a considerable degree of nonstationarity probably due to ram pressure variantions in the solar wind, a supersonic signature of the ions on the downstream side of the shock, and multiple shock crossings, with $x_{\mathrm{VOY}-2, \min } \simeq 1.6$ (Richardson et al. 2008).

Comparable nonstationary behaviours are also observed by the Cluster experiment at the Earth bow shock, which is even more turbulent and nonstationary than the plasma reflected in Voyager data (see e.g. Lobzin et al. 2007). Therefore, to fully understand small-scale observational behaviour, one requires a theory describing the emergence of unstable plasma distribution functions and subsequent turbulence generation at the shock. The turbulence description most commonly found in plasma literature is based on a plasma wave approach (i.e. a Fourier transform) of the dynamical parts of the particle distribution function $f(\boldsymbol{x}, \boldsymbol{v}, t)$ and the fields, i.e. from this point of view, turbulence is the macroscopic, overall behaviour of many superimposed microscopic plasma waves.

In an MHD shock, these waves need to be described consistently with the jump conditions. In literature, only a few similar approaches may be found, among the earliest studies partially comparable with the direction into which this study is aiming (e.g. McKenzie \& Westphal 1969; Chao \& Goldstein 1972). McKenzie \& Westphal (1969) studied, theoretically, the transport of upstream Alfven waves being transported across the shock, while Chao \& Goldstein (1972), allowing for fluctuating plasma quantities, also reconstructed the shock normals of various spacecraft shock crossings from experimental data. Introducing fluctuating MHD quantities as a Fokker-Planck-like approach (where e.g. $\boldsymbol{B}=\boldsymbol{B}_{0}+\delta \boldsymbol{B}(\boldsymbol{x}, t)$ ), the latter authors even provide a description of anisotropic pressures, although tied to the WKB-convection of $\delta \boldsymbol{B}$. In contrast to these earlier publications, the present study aims to describe not only the convection of MHD waves across the shock, but also the generation of turbulent energy by wave excitation through free thermal energy created at the shock, complemented by a pressure anisotropy which is not tied to plasma waves. 
A consistent, general description of plasma wave generation requires a kinetic approach to the system, while MHD is a representation of the kinetic distribution function by its lowest order velocity moments only. While many time-dependent extensions to MHD exist, no systematic and self-consistent approach for turbulence under arbitrary conditions is available. Among the theoretical approaches to MHD turbulence in astrophysical shocks, one finds e.g. Krasnoselskikh et al. (2002), who performed numerical studies of MHD shocks in one dimension, describing the quasi-perpendicular shock as a superposition of Whistler waves. Lembege et al. (2004) studied multiple unresolved topics of the shock transition such as the problems already mentioned earlier in this text, including shock front nonstationarity, electron heating, and particle diffusion in turbulent media, using theoretical and numerical methods. Liu et al. (2007) did use an analytical approach based on the plain anisotropic MHD jump conditions (Hudson 1970), where the mirror and firehose instabilities as well as an entropy argument are applied to estimate the overall stability of the downstream plasma, as a function of the local upstream MHD conditions and the shock density compression ratio. A similar approach was taken by Fahr \& Siewert (2007, 2009), who completed the MHD jump conditions using a dissipation-free kinetic model based on kinetic invariants (related to results by Chew et al. 1956) to estimate the amount of free energy available for conversion into non-classical degrees of freedom (i.e. plasma waves), based on the MHD formulation of the mirror mode instability condition. Finally, Verscharen \& Fahr (2008) based their studies on a kinetic model including particle-wave interactions at a parallel field orientation, which appeared in the solar wind termination shock during a polarity change in the solar magnetic field, temporarily overriding the default Parker spiral field (Parker 1958). Despite the different modelling approaches, the main point of all these studies is that plasma waves need to be introduced to describe the non-ideal behaviour observed in astrophysical shocks. In this study, we expand upon the results obtained by Fahr \& Siewert (2007, 2009), repeating their studies of the quasi-perpendicular shock, but now for a quasi-parallel magnetic field orientation.

The onset of the above mentioned plasma instabilities is triggered by an over-critical ion pressure anisotropy $\lambda=p_{\perp} / p_{\|}$, which, for further studies, needs to be extracted either from adequate shock data or from the appropriate Rankine-Hugoniot MHD jump conditions. However, deriving this parameter from jump conditions is complicated by the fact that, as pointed out by Erkaev et al. (2000), Vogl et al. (2003) and Liu et al. (2007), the classical formulation of the MHD jump conditions for anisotropic pressures (Hudson 1970) is underdetermined, leaving only 6 equations to determine 7 downstream parameters as a function of the upstream parameters. Among the analytical approaches found in literature, even the choice of the free parameter is not unique. For example, Erkaev et al. (2000) selected the downstream pressure anisotropy $\lambda_{2}$ for their parameter study, while Liu et al. (2007) selected the mass compression ratio $x$. Then, using experimental data acquired by the Voyager-1 spacecraft at the solar wind termination shock, Liu et al. (2007) were able to fix the unknown downstream compression ratio and therefore close the system of equations numerically for the perfectly parallel and perpendicular shocks. However, this is an unsatisfying approach, since it does not in principle allow us to close uniquely the jump conditions. Instead, one requires a systematical, theoretical approach that provides a sufficiently general expression complementing the classical jump conditions. Having realised this problem, we derived a self-consistent, basic kinetic approach to the MHD shock (Fahr \& Siewert 2006; Siewert \& Fahr 2008), describing the ion passage over a shock transition layer by solving Boltzmann-Vlasow equations based on the conservation of CGL invariants (Chew et al. 1956). This approach is able to predict the downstream pressure anisotropy for arbitrary upstream conditions, offering the additional pieces of information necessary (but insufficient) to close the jump conditions, and allowing us to understand qualitatively the generation of turbulence based on an MHD instability condition, such as those given by Erkaev et al. (2000). This approach does not yet allow a description of the wave power spectrum.

In this study, we use these results to investigate the density compression ratios $x \leq x_{\text {crit }}$ allowed for subcritical shocks that do not generate instability-driven turbulences and for supercritical shocks with $x \geq x_{\text {crit }}$ driving such turbulences. This approach is well supported by studies performed by both Liu et al. (2007) and Génot (2008), who also performed a stability study, although based on Voyager data, complementing the incomplete jump conditions. In contrast to these earlier studies, our kinetic model allows us to at least conceptually close the system of jump conditions, due to the system ending up potentially overdetermined. We focus on the parallel shock, which, in the solar system, is found at planetary bowshocks and prevails at the termination shock during current-sheet passages. Studies of a parallel MHD shock carried out on the basis of Cluster data can also be found in literature (see Lucek et al. 2007).

\section{The way to close the parallel jump conditions}

The classical MHD jump conditions for a pressure-anisotropic shock first were derived by (Hudson 1970). They consist of six conservation equations, of which, in this study, we only consider the energy flow conservation,

$$
\begin{aligned}
& {\left[\left[U_{n}\left(\frac{1}{2} p_{\|}+2 p_{\perp}+\frac{\rho U^{2}}{2}\right)+\frac{B_{n}^{2} U_{n}}{B^{2}}\left(p_{\|}-p_{\perp}\right)\right.\right.} \\
& \left.\left.\quad+\frac{B_{n} \boldsymbol{B}_{t} \cdot \boldsymbol{U}_{t}}{B^{2}}\left(p_{\|}-p_{\perp}\right)+\frac{\boldsymbol{B}_{t}}{4 \pi} \cdot\left(U_{n} \boldsymbol{B}_{t}-\boldsymbol{U}_{t} B_{n}\right)\right]\right]=0 .
\end{aligned}
$$

As mentioned earlier, one needs to select one of the seven downstream parameters $\left(\rho, U_{n, t}, B_{n, t}\right.$, and $p_{\|, \perp}$ on the downstream side), which must be fixed using an independent physical relation.

Faced with this problem, we developed a kinetic formalism (Siewert \& Fahr 2008), which is able to predict the following relations between the upstream and downstream pressure components if applied to the parallel shock,

$p_{\|, 2}=x^{3} p_{\|, 1} \quad p_{\perp, 2}=x p_{\perp, 1}$.

However, this introduces two equations where only one is required, potentially leading to an overdetermined system of equations, unless it is possible to prove that two of these equation are mathematically equivalent. In addition, a solution of the jump conditions still requires numerical methods. The only viable possibilities for perfectly closing the jump conditions would be either to introduce an additional downstream parameter, or eliminate one of the eight equations. Both of these possibilities require an extended physical model of the shocked system, introducing additional physical processes.

For a parallel shock, the simplest argument for this requirement is that the upstream system consists of a plasma moving parallel to a quiet background magnetic field, which, without superimposing hydromagnetic waves, does not induce "magnetic" decelerations. To explain this, one requires an electric 
field ramp decelerating the protons. This ramp, however, would lead to acceleration of the electrons that are carrying an opposite charge. The resulting system would produce a global wavelike behaviour, asymptotically returning to a wave-free plasma flow on the downstream side (see Verscharen \& Fahr 2008). To explain the bulk deceleration, some of the particle kinetic energy must therefore be dissipated into different degrees of freedom, such as local plasma waves.

The most basic approach to adding plasma wave terms to the MHD jump conditions follows from the following assumptions, and that the wave power distribution function does not contribute to the overall momentum flow. The total wave energy is therefore the only modification that must be taken into account by the MHD jump condition describing the energy conservation, for which we make a simple approach in the form

$\left[\left[W_{\text {plasma }}+W_{\text {waves }}\right]\right]=0$.

Thus, without any additional assumptions, the expression $W_{\text {waves }}$ is a new parameter entering the equations that allows to close the system of jump conditions.

It should be noted that the specific type of waves being generated has not been specified yet; instead, we just use a placeholder for the wave energy which is used to close the system of jump conditions. In Sect. 4, we present a simple approach to deriving a wave energy term at the parallel shock, and how this approach may be used to obtain an additional free parameter to close the jump conditions.

\section{The condition for firehose-induced plasma wave generation}

In this section, we prepare the derivation of the plasma wave term by studying the firehose condition in the analytical framework of the kinetic model. First, we note that Siewert \& Fahr (2008) demonstrated that a parallel shock will always reach a smaller downstream pressure anisotropy (defined by $\lambda=p_{\perp} / p_{\|}$) compared to its upstream value, expressed by (see Eq. (2))

$\lambda_{2}=\frac{1}{x^{2}} \lambda_{1}$

i.e. $\lambda_{2}<\lambda_{1}$. Now, it is known that a pressure anisotropy of this type may trigger the firehose instability, which appears for (Thompson 1964),

$\lambda_{2} \leq 1-\frac{2}{\beta_{\|, 2}}$

in the MHD limit. Here, $\beta_{\|, 2}$ is the usual plasma beta, for which the analytical solution presented in the last section allows us to derive a similar upstream-to-downstream relation

$\beta_{\|, 2}=\frac{p_{\|, 2}}{\frac{B_{2}^{2}}{8 \pi}}=\frac{x^{3} p_{\|, 1}}{\frac{B_{1}^{2}}{8 \pi}}=x^{3} \beta_{\|, 1}$.

Using Eq. (4), the firehose condition (Eq. (5)) may now be written in the form

$\lambda_{2}=\frac{\lambda_{1}}{x^{2}} \leq 1-\frac{2}{x^{3} \beta_{\|, 1}}$,

This equation may be brought into the form

$x^{3}-x \lambda_{1} \geq \frac{2}{\beta_{\|, 1}}$.

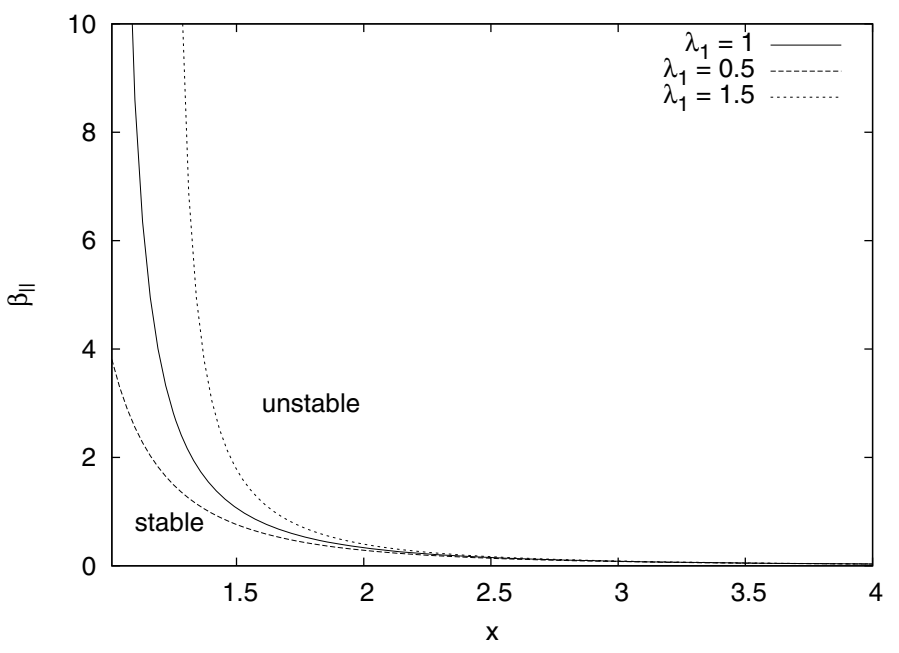

Fig. 1. Stable and unstable regions of a parallel MHD shock in the $\beta_{\|, 1}-x$ space for different $\lambda_{1}$.

This expression allows us to estimate, as a function of the compression ratio $x$ (other than the upstream parameters), whether the firehose instability emerges or not. First, we see that, for $\lambda_{1}>1$, the left side of the inequality becomes negative (where $x^{2}<\lambda_{1}$ ) and an absolutely stable region emerges in the weak shock region, where the compression $x$ is close to unity. For $\lambda_{1} \leq 1$, however, any shock may become unstable, depending on the upstream parameters (see Fig. 1, where we plotted the value $x_{\text {crit }}$, corresponding to the identity in the above equations).

Equation (8) likewise proves that, given an MHD compression ratio of $x$, the plasma becomes unstable at high plasma beta values. Rewriting $\beta_{\|, 1}$ in terms of Mach numbers, we obtain

$\beta_{\|, 1}=2 \frac{M_{\mathrm{A}, 1}^{2}}{M_{\mathrm{s}, \|, 1}^{2}} \quad$ and $\quad x^{3}-x \lambda_{1} \geq \frac{M_{\mathrm{s}, \|}^{2}}{M_{\mathrm{A}}^{2}}$.

This means that a system is firehose-unstable when the Alfvenic Mach number $M_{\mathrm{A}}$ is much higher than the parallel sonic Mach number $M_{\mathrm{s}, \|}$. In other words, strong sonic shocks are firehose-stable, and strong magnetosonic shocks are firehoseunstable. This result is unsurprising, because it is well known that MHD instabilities require a strong magnetic field to trigger the instability, whereas we have now derived a quantitative formulation of this relation for MHD shock waves.

\section{The energy transfer between kinetic and wave degrees of freedom}

Finally, we repeat the study performed by Fahr \& Siewert (2007) and derive the energy transferred into the wave degrees of freedom. Following the earlier approach, we assume that only the parallel pressure component is modified by the wave generation in the firehose-unstable case, i.e.

$\Delta E=\frac{\Delta W}{U_{n}}=\frac{1}{2}\left(p_{\|, 2}-\tilde{p}_{\|, 2}\right)=\frac{1}{2}\left(x^{3} p_{\|, 1}-\tilde{p}_{\|, 2}\right)$,

where, using the same approach as in the last section, we have assumed that the internal parallel energy $\left(\propto 1 / 2 p_{\|}\right)$is converted into wave energy. The marginally stable downstream pressure derived from Eq. (9) is found to be $\tilde{p}_{\|, 2}$, when freezing all other 
parameters at their classical, wave-free downstream values. An analytical expression of this value may be derived from Eq. (5),

$\tilde{p}_{\|, 2}=p_{\perp, 2}-\frac{B_{1}^{2}}{4 \pi}=x p_{\perp, 1}-\frac{M_{\mathrm{s}, \|, 1}^{2}}{M_{\mathrm{A}, 1}^{2}} p_{\|, 1}$.

This means that the internal energy transferred into plasma waves is

$\Delta E=\frac{1}{2}\left(p_{\|, 2}-\tilde{p}_{\|, 2}\right)=\frac{1}{2}\left(\frac{M_{\mathrm{s}, \|, 1}^{2}}{M_{\mathrm{A}, 1}^{2}} p_{\|, 1}+x^{3} p_{\|, 1}-x p_{\perp, 1}\right)$.

Given an observed wave field on the downstream side of a parallel shock, this equation allows us to estimate the MHD compression ratio numerically (or vice versa) and might therefore be useful for studies of astrophysical shocks.

However, we need to point out that the approach presented here was based on the assumption that only the internal energy stored in the parallel component is modified by the wave field, leaving the other terms proportional to $p_{\|}$untouched. In addition, using Eq. (12) in addition to the modified jump conditions again leads to an overdetermined system of equations, with $\Delta E$ (respectively $\Delta W$ ) being eliminated again from the equations. Verifying that the resulting equations are selfconsistent is, again, very complicated and needs to be perfomed in a systematical way, allowing for a wider variety of wave generating mechanisms. For example, one possible way to do this would be to ignore Eq. (12) and use $\tilde{p}_{\|, 2}$ as an additional free parameter, which, physically, corresponds to an "unknown" wave energy generation process modifying the system. Since other parameterisations and model approaches are possible as well, a detailed study of the method outlined here is clearly beyond the scope of this letter, and probably also beyond the scope of any individual research paper.

\section{Conclusions}

We have presented a possible method for closing the anisotropic jump conditions describing a parallel MHD shock. This method uses a simple particle-wave interaction mechanism in the MHD approximation, which manifests itself as a modification of the jump conditions. This approach allows us to present a method for closing the otherwise underdetermined system of anisotropic jump conditions.

Using the MHD firehose instability condition, we demonstrated how a specific physical process may be added to the jump conditions in an explicit way. This approach allows us to give an analytical form of the marginally stable parallel pressure, and the amount of energy that is exchanged with the plasma wave component as well. Although variations in this model approach do exist, we have demonstrated that a pressure-isotropic upstream plasma flow will remain stable on the downstream side only when the shock is weakly magnetosonic.

\section{References}

Burlaga, L. F., Ness, N. F., Acuna, M. H., et al. 2005, Science, 309, 2027 Burlaga, L. F., Ness, N. F., \& Acuna, M. H. 2006, Geophys. Res. Lett., 33, L21106

Burlaga, L. F., Ness, N. F., Acuna, M. H., et al. 2008, Nature, 454, 75

Chao, J. K., \& Goldstein, B. 1972, J. Geophys. Res., 77, 5455

Chew, G. F., Goldberger, M. L., \& Low, F. E. 1956, Proc. R. Soc. London A, 236, 112

Decker, R. B., Krimigus, S. M., Roelof, E. C., et al. 2005, Science, 309, 2020

Decker, R. B., Krimigus, S. M., Roelof, E. C., et al. 2008, Nature, 454, 67

Diver, D. A. 2001, A Plasma Formulary for Physics, Technology and Astrophysics (Hoboken, New Jersey: John Wiley)

Erkaev, N. V., Vogl, D. F., \& Biernat, H. K. 2000, J. Plasma Phys., 64, 561

Fahr, H.-J., \& Siewert, M. 2006, A\&A, 458, 13

Fahr, H.-J., \& Siewert, M. 2007, ASTRA, 3, 21

Fahr, H.-J., \& Siewert, M. 2009, ApJ, 693, 281

Fisk, L. A. 2005, Science, 309, 2016

Génot, V. 2008, ApJ, 687, L119

Gombosi, T. I. 1998, Physics of the Space Environment (New York: Cambridge University Press)

Gurnett, D. A., \& Kurth, W. S. 2005, Science, 309, 2025

Gurnett, D. A., \& Kurth, W. S. 2008, Nature, 454, 78

Hudson, P. D. 1970, Planet. Space Sci., 18, 1611

Jokipii, J. R. 2008, Nature, 454, 38

Kiraly, P. 2005, in Proc. 29th Internat. Cosmic Ray Conference, ed. B. S. Acharya, S. Gupta, P. Jagadeesan, et al., 1, 339

Krasnoselskikh, V. V., Lembege, B., Savoni, P., \& Lobzin, V. V. 2002, Phys. Plasmas, 9, 1192

Lembege, B., Giacalone, J., Scholer, M., et al. 2004, Space Sci. Rev., 110, 161

Liu, Y., Richardson, J. D., Belcher, J. W., \& Kasper, J. C. 2007, ApJ, 659, L65

Lobzin, V. V., Krasnoselskikh, V. V., Bosqued, J.-M., et al. 2007, Geophys. Res. Lett., 34, L05107

Lucek, E. A., Horbury, T. S., Dandouras, I., \& Reme, H. 2007, J. Geophys. Res., 113, A07S02

McKenzie, J. F., \& Westphal, K. O. 1969, Planet. Space Sci., 17, 1029

Parker, E. N. 1958, ApJ, 128, 664

Richardson, J. D., Kasper, J. C., Wang, C., Belcher, J. W., \& Lazarus, A. J. 2008, Nature, 454, 63

Siewert, M., \& Fahr, H.-J. 2008, A\&A, 485, 327

Stone, E. C., Cummings, A. C., McDonald, F. B., et al. 2008, Nature, 454, 71

Stone, E. C., Cummings, A. C., McDonald, F. B., et al. 2005, Science, 309, 2017

Thompson, W. B. 1964, An Introduction to Plasma Physics (New York: Pergamon)

Verscharen, D., \& Fahr, H.-J. 2008, A\&A, 487, 723

Vogl, D. F., Langmayr, D., Erkaev, N. V., et al. 2003, Planet. Space Sci., 51, 715 